\title{
Strates
}

STRATES Matériaux pour la recherche en sciences sociales

Hors-série | 2002

Parcours dans la recherche urbaine, Michel Rochefort, un géographe engagé

\section{Michel Rochefort et la question urbaine dans le Tiers-Monde}

\section{Alain Durand Lasserve}

\section{OpenEdition}

\section{Journals}

Édition électronique

URL : http://journals.openedition.org/strates/558

DOI : $10.4000 /$ strates.558

ISSN : 1777-5442

Éditeur

Laboratoire Ladyss

Édition imprimée

Date de publication : 1 janvier 2002

ISSN : 0768-8067

Référence électronique

Alain Durand Lasserve, « Michel Rochefort et la question urbaine dans le Tiers-Monde », Strates [En ligne], Hors-série | 2002, mis en ligne le 18 mai 2005, consulté le 08 septembre 2020. URL : http:// journals.openedition.org/strates/558; DOI : https://doi.org/10.4000/strates.558

Ce document a été généré automatiquement le 8 septembre 2020

Tous droits réservés 


\title{
Michel Rochefort et la question urbaine dans le Tiers-Monde
}

\author{
Alain Durand Lasserve
}

1 Comment as-tu été conduit à travailler sur les villes du Tiers-Monde et en quoi cela a-t-il influencé ton approche de la question urbaine?

2 Il faut rappeler que j'ai d'abord été formé par une réflexion sur la ville, l'aménagement du territoire et les métropoles d'équilibre en France. Jusqu'au milieu des années soixante, exception faite du petit livre d'Yves Lacoste, relativement peu de travaux de géographes avaient abordé la question du sous-développement en tant que telle.

3 J'ai d'abord découvert ce qu'étaient les villes des pays dits sous-développés à l'occasion d'un long voyage que j'ai fait en 1956 à travers le Mexique, le Nicaragua, l'équateur et le Pérou, pour arriver au Brésil. Ce que j'ai observé m'a conduit à réfléchir sur mon approche de la ville, fondée jusque là sur ma pratique de la recherche sur les villes françaises. C'était un peu une découverte. Mais j'en suis resté là.

4 C'est quelques années après, lorsque je suis allé au Brésil en 1960-1961, que j'ai repris cette réflexion engagée cinq ans auparavant. Ce second séjour en Amérique latine a d'abord été une découverte de la réalité brutale, celle de la pauvreté urbaine des bidonvilles le long de la Reforma de Mexico, des barriadas de Lima et des favelas de Rio de Janeiro. Mais il a constitué le point de départ d'une réflexion plus systématique sur les facteurs de différenciation entre les villes des pays développés et celles des pays du Tiers-Monde. Il y avait là un champ de recherche considérable pour la géographie française. Lorsque je suis entré au comité technique de géographie de l'Orstom en 1967, puisque l'on m'a confié la présidence du comité technique d'économie, j'ai eu l'occasion de découvrir ce qu'était la réalité des pays d'Afrique sub-saharienne. Parallèlement, lorsque j'ai été nommé à Paris en 1964, j'ai commencé une collaboration avec le Maroc et la Tunisie. En 1968, j'ai découvert l'Inde ; cela a été très important pour moi. Pendant toutes ces années - et je le regrette un peu aujourd'hui - je n'ai toutefois fait, dirigé ou impulsé des recherches que sur le Brésil. J'y ai apporté des méthodes d'analyse de la géographie urbaine française ainsi que mes propres méthodes d'étude du secteur tertiaire, des réseaux et des systèmes urbains. J'ai certes contribué à lancer des études 
brésiliennes sur les villes et l'aménagement régional. Je dois aussi admettre que certaines de ces recherches, très inspirées par une méthodologie mise au point dans le contexte français étaient trop détachées du contexte brésilien.

6 Non, mais ça en a peut-être été une pour les Brésiliens, un élément perturbateur par rapport à leurs propres réflexions sur la réalité de la formation sociale brésilienne un peu trop laissée dans l'ombre. Cela a été corrigé par la suite mais, à cette époque là, les premiers enseignements au Brésil portaient certainement trop sur ce que j'appelle la géographie française et la " méthodologie Rochefort » alors que, confronté à une réalité différente, il eût fallu adopter une démarche et des méthodes de recherche différentes de celles qui étaient privilégiées à partir de l'expérience française. Il s'agit de la première phase.

7 La fin des années 1960 va être marquée par un profond bouleversement qui marquera durablement mon approche du Tiers-Monde. La question de l'impérialisme et de l'opposition fondamentale entre des pays dominants et des pays dominés émerge au premier plan du débat sur le développement. Les écrits de Gunder Frank et de Samir Amin contribuent à ce renouvellement de la réflexion sur les rapports entre domination et sous-développement. Dans ce contexte, l'analyse du tertiaire constituait un élément clef dans l'étude de l'impact des rapports de domination et de leurs effets sur les problèmes urbains. Cela a eu une influence décisive sur mon enseignement et mes directions de thèses. L'une des questions centrales a été d'évaluer systématiquement, les spécificités des systèmes urbains des pays du Tiers-Monde en fonction du problème de la domination, l'hypothèse de départ étant que la plupart des systèmes urbains des pays du Tiers-Monde ont, à un certain moment de leur histoire, été créés par l'extérieur, pour l'extérieur et ne sont pas le produit d'une dynamique interne de la société dans laquelle ils se trouvent. Plusieurs travaux de thèse ont repris cette hypothèse de travail et l'ont développée, aussi bien pour l'étude des systèmes urbains que pour celle de l'organisation de l'espace urbain. Je pense que le meilleur exemple, en France, d'une telle approche - et le travail le plus abouti - est la thèse de Signoles sur Tunis et l'espace tunisien. J'ai également dirigé de nombreuses thèses dans les pays du Tiers-Monde (rien qu'au Brésil j'ai dirigé 38 thèses qui ont abouti à des doctorats), donc c'était toujours un petit peu mon leitmotiv. L'une des dernières thèses que j'ai dirigée et qui reprend cette hypothèse est celle de Sabah Abouessalam ${ }^{1}$ sur Pauvreté urbaine et comportements résidentiels à Marrakech.

Avec le recul, quel regard critique portes-tu sur cette hypothèse et tes choix méthodologiques?

9 Mon apport personnel à la réflexion c'est, au départ, mon travail sur les réseaux urbains et les métropoles d'équilibre. Par la suite, en particulier sur le Tiers-Monde, j'ai intégré et interprété les idées des autres, y compris celles des étudiants dont j'ai dirigé la thèse, mais j'ai moi-même peu écrit. J'ai, pour l'essentiel, enseigné et dirigé des recherches. Celles-ci reflètent bien le courant de pensée - disons marxien - auquel je me rattachais alors. Il faut bien admettre que nous avons sans doute surestimé la question de la domination. Nous avons ainsi trop systématiquement occulté le poids des différences socio-culturelles dans les pays en développement. La question de la domination restait très marquée par l'analyse que nous faisions du rôle de l'impérialisme, y compris dans notre analyse des villes. J'ai, par la suite, dans le courant des années 1980, essayé de corriger cette approche trop systématique en insistant sur la question des spécificités et des caractères particuliers des sociétés du Tiers-Monde. Si la 
question de l'impérialisme et de la domination reste centrale, elle ne me semble plus constituer une hypothèse de travail fondamentale. S'interroger aujourd'hui sur la spécificité de l'impérialisme me semble d'un intérêt limité. On en a dit assez sur l'impérialisme.

Ce qui nous intéresse maintenant, ce sont les effets de cette domination compte tenu des traditions antérieures ou des capacités de réaction des sociétés concernées. Cette interrogation soulève un ensemble de questions d'ordre social, économique et culturel. Une telle approche ne relève pas seulement de la géographie. Je préfère parler d'études urbaines.

11 Je dirais qu'aujourd'hui, j'attache beaucoup d'importance à la question de la spécificité des sociétés du Tiers-Monde et en particulier des sociétés urbaines. Dans le même temps, je me méfie comme de la peste, des deux mots-clés "mondialisation» et "globalisation ». Je m'explique : ces deux termes reflètent un phénomène indiscutable et de grande ampleur qui touche aux mutations profondes de la structure productive capitaliste, à l'organisation des échanges, donc des flux et à ses conséquences sur les villes, sur la situation de l'emploi et la flexibilité, sur les inégalités sociales et sur la société urbaine en général. Nous sommes ici confrontés à une forme nouvelle et peutêtre plus accentuée de domination d'un petit nombre de pays sur tous les autres pays de la planète. Dans un sens, la question de la mondialisation nous renvoie à celle d'une nouvelle forme d'impérialisme, dont la stratégie des très grandes entreprises et les flux qu'elles contrôlent ou organisent dessine les contours.

12 Leur impact sur les villes est considérable, que ce soit par l'utilisation qu'elles font de la main-d'œuvre bon marché dans les pays du Tiers-Monde - les implantations se font sur la pauvreté ce qui implique qu'on la maintienne - ou par la mise en place de relais de domination qui prennent appui sur des métropoles relais, dépendantes. Il y a là des différences fondamentales entre les villes du Tiers-Monde et celles des quelques grands pays qui s'adjugent l'avantage de la mondialisation-globalisation. Derrière ces mots qui sont des mots traitres pour moi, je pense que se cachent de nouvelles formes de domination qui pèsent, en bien ou en mal, sur les villes du Tiers-Monde. On assiste à l'émergence d'un nouveau tertiaire au service des entreprises. C'est lui qui, à mon avis, va différencier les grandes métropoles dominantes des villes et métropoles du TiersMonde. Le nouveau tertiaire qu'on connaît bien dans les grandes métropoles développées, on le retrouve dans le Tiers-Monde sous des formes différentes : soit celle d'entreprises de sous-traitance, par exemple pour les grandes entreprises tertiaires informatiques des pays développés, soit celle d'entreprises indépendantes. Mais cellesci, créées souvent avec l'aide de capitaux étrangers, sont au service de la stratégie des grandes entreprises multinationales dont les sièges sont dans les pays développés. Ce nouveau tertiaire est sans aucun doute intéressant pour les pays sur le plan de l'emploi et contribue donc à l'augmentation de la richesse, mais il interdit le développement autonome de l'économie nationale. Il ne s'agit pas d'un tertiaire qui se développerait en fonction des conditions et des formes de développement des économies nationales concernées.

13 Peut-on encore parler aujourd'hui de "développement autonome ", voire de "développement autocentré»?

Quand je parle de "développement autonome", je pense à un développement qui viserait à valoriser les capitaux nationaux, en particulier dans les secteurs industriel et agricole. Nous sommes dans une situation où les pays du Tiers-Monde s'industrialisent 
à coup de capitaux étrangers et en fonction de la stratégie des grandes entreprises des pays dominants sur le plan mondial. Dans ce contexte, le nouveau tertiaire qui se développe peut être indépendant, ce qui est positif, mais il est d'abord au service d'une stratégie étrangère, ce qui l'est moins. Je pense que ce tertiaire n'est pas au service d'un développement plus "autocentré ». Ce terme pose problème, en particulier lorsqu'on parle de la production. Aujourd'hui, exception faite des héritages des formes antérieures de petites productions marchandes ou de petites paysanneries, une activité productive, qu'elle soit agricole ou industrielle, est obligatoirement ouverte sur le monde. Les échanges sont mondialisés, il faut donc s'y adapter. Mais cette adaptation pourrait être le fait d'entreprises qui, étant à capitaux nationaux, seraient génératrices d'accumulation de capital susceptible d'être réinvesti dans le pays et donc de générer un certain processus qu'on peut appeler développement (du moins on peut l'espérer car le capitalisme même national, peut toujours aller investir ailleurs). Quand je parle de développement autocentré, je me réfère à la propriété du capital, non pas à une production destinée au marché national.

$15 \mathrm{Si}$, au contraire, les capacités productives du pays sont entre les mains de grandes entreprises étrangères qui y valorisent leurs capitaux directement, il est évident que leur stratégie n'a que faire des projets de développement du pays. Elles restent là tant que c'est intéressant. Quand ça ne l'est plus, en particulier si les salaires augmentent, elles trouvent un autre endroit où les salaires sont moins élevés. Dans ce contexte, l'accumulation de capital n'est pas un phénomène qui va permettre le développement de l'économie du pays.

16 Le nouveau tertiaire qui se développe dans les plus grandes villes des pays du TiersMonde est la conséquence de la mondialisation. Cette dynamique ne semble pas réversible. Comment, dans ce contexte, concevoir les effets de la mondialisation dans les villes et particulièrement dans les grandes villes du Tiers-Monde. L'opposition qui se précise aujourd'hui entre quelques grands pays dominants et des pays dominés correspond-elle toujours aux catégories "pays développés » et "Tiers-Monde »? Rien n'est moins sûr. La mondialisation a et aura sans doute un impact de plus en plus important sur les différenciations entre grandes villes. On parle beaucoup de la nouvelle métropolisation: elle est indiscutable dans les grandes villes des pays développés. Mais il y a une autre forme de métropolisation, qui aboutit aussi à la croissance des plus grandes villes dans les pays du Tiers-Monde selon des modèles et des mécanismes qui ne sont pas ceux des années 1970 et 1980. Ce n'est plus tout à fait la même forme de métropolisation. On ne peut donc utiliser le même terme de métropolisation pour parler de deux réalités différentes. Il faudrait recourir à deux termes différents. Il y a une métropolisation dominante et une métropolisation dominée.

17 Qui considères-tu comme les principaux acteurs de la domination: les grandes entreprises et groupes multinationaux ou les États?

18 La mondialisation cache une nouvelle domination. Si l'on étudie les formes de cette domination sur les villes et particulièrement les grandes villes, on voit qu'elle est avant tout, au départ, le résultat de la stratégie des grandes entreprises multinationales. Qui sont, alors, les acteurs de la domination? Quelle est l'attitude des États, des pouvoirs nationaux ou locaux par rapport à ces stratégies? Ce qui m'intéresse c'est la capacité (ou l'incapacité) de ces pouvoirs à harmoniser leur propre stratégie avec celles des grandes entreprises multinationales, pour les attirer et bénéficier de leurs choix de 
localisation. Cela vaut aussi bien pour Lyon que pour Tunis. C'est un facteur très important de différenciation entre les villes.

Dans certains pays capitaux nationaux et entreprises nationales - il en existe encore peuvent contrebalancer les stratégies des entreprises multinationales. Ceci ne doit pas non plus être sous-estimé. Je distingue bien la mondialisation des échanges de la nationalité du capital. Il peut y avoir des capitalismes nationaux qui continuent de bénéficier de l'aide des pouvoirs nationaux, et qui ont une certaine marge de manœuvre par rapport aux grandes entreprises multinationales. Par exemple, la situation de São Paulo est différente de celle d'Abidjan. Si ce ne sont plus à proprement parler des États qui dominent mais des grandes entreprises mondialisées, les directions et centres de décisions restent malgré tout dans un petit nombre de grands pays. Il y a quand même une localisation.

$T u$ estimes que la compétition que se livrent les grandes métropoles du monde pour attirer les capitaux multinationaux tend à accélérer encore le processus de différenciation qui existe entre les villes qui font partie de ce grand maillage mondial et les villes qui sont laissées un peu à l'écart. Dans ce contexte là, comment vois-tu l'Afrique?

21 Je la connais peu maintenant : mes derniers séjours en Afrique remontent à quelques années, mais je la vois mal partie. Un des éléments clés dans la stratégie des grandes entreprises, c'est la pauvreté parce que cela permet d'avoir une main-d'œuvre bon marché. Or, la pauvreté asiatique est quand même beaucoup plus « intéressante » que la pauvreté africaine. La chance de l'Afrique serait que la pauvreté asiatique diminue et que donc, les salaires augmentent. En raison du coût de sa main-d'œuvre, l'Afrique deviendrait alors attractive pour les investisseurs. Reste le problème de la qualification de cette main-d'œuvre.

22 La situation du Maghreb est très différente de celle de l'Afrique sub-saharienne. Les pays du Maghreb ont le vent en poupe, ils ont des traditions. Il leur manque toutefois la capacité d'investissement au niveau national et leur expérience de la production moderne n'est sans doute pas suffisante. Il y a donc un risque que, malgré leurs atouts, ils ne tirent pas profit des opportunités qui peuvent se présenter.

23 Comment vois-tu dans cette nouvelle redistribution, le rôle de la Banque mondiale et des organisations du système des Nations-Unies, je pense en particulier au CNUEH-Habitat, et au PNUD. Peuvent-elles faire autre chose qu'accélérer le processus de polarisation en cours? Peuvent-elles avoir un effet modérateur en essayant d'en freiner les effets les plus négatifs en particulier au niveau social?

24 La mondialisation tend à accentuer les inégalités. Or les grandes entreprises multinationales ne peuvent se permettre de pousser le cynisme trop loin. Nous ne sommes plus en Alsace à la fin du XIX siècle. La misère était horrible, mais les entrepreneurs pouvaient se permettre de trouver que la vie était comme ça.

Aujourd'hui, les grandes entreprises, quelle que soit leur puissance financière, ont à compter avec des États qui représentent des sociétés. Tout n'est pas permis et les firmes doivent veiller aux conséquences négatives de leurs stratégies sur les masses urbaines. Il faut donc qu'il y ait des instances ou des mécanismes qui visent à réduire les inégalités sociales mais surtout leurs effets, diminuant ainsi les risques de tensions sociales susceptibles de compromettre l'intérêt des grandes entreprises capitalistes. De la même manière qu'à une certaine époque le rôle de l'aménagement du territoire en France était de diminuer les effets négatifs des choix économiques pour éviter les 
tensions sociales, il y a aujourd'hui, à l'échelle mondiale, des organismes qui vont essayer d'aider les pays et surtout les villes - c'est important pour nous - à diminuer objectivement les conséquences des inégalités sociales, en particulier sur l'habitat. Ceci explique en partie que, malgré tout ce que je viens de dire sur l'accentuation des inégalités, le bilan des quarante dernières années dans les pays du Tiers-Monde montre une certaine amélioration de la situation du logement. Il y a une très forte augmentation de l'espace utilisé par les pauvres, mais une certaine amélioration de ces espaces de pauvreté et, d'une façon globale, une certaine diminution de l'extrême précarité. La situation n'est bien sûr pas la même selon les régions et les villes. Par exemple, l'amélioration de la situation est plus sensible dans les espaces de pauvreté de Rio de Janeiro que d'Abidjan ou de Bombay. En Amérique du sud on ne peut nier qu'il y ait une aggravation relative des inégalités sociales : les riches deviennent plus riches et on observe une prolifération des espaces de pauvreté en milieu urbain, accompagnée par une différenciation de ces espaces les uns par rapport aux autres. Mais on observe dans le même temps une diminution, voire une disparition, des espaces de très grande précarité.

26 La situation sociale des pauvres des villes s'est-elle aggravée ou plutôt améliorée au cours des quatre dernières décennies? Qu'en est-il de la mobilité sociale?

27 C'est une question que je me pose mais je n'ai jamais vu d'études globales suffisamment approfondies sur ce sujet pour pouvoir répondre avec certitude. Honnêtement, je ne sais si l'on peut dire que la situation s'est globalement améliorée. J'ai un certain nombre d'exemples, mais au niveau de biographies ou d'histoires familiales, d'ascensions familiales modestes. J'ai également des exemples inverses de chute dans la pauvreté de gens qui appartenaient aux groupes que l'on peut appeler moyensinférieurs. Je ne peux toutefois généraliser. Il y a de nombreux exemples de mobilité, en particulier dans les études sur le secteur informel, mais je ne saurais dire si c'est mieux ou plus facile maintenant qu'avant. Une chose est certaine: quantitativement, le nombre de pauvres urbains est en augmentation, puisqu'on a un accroissement global de la population des villes et que le pourcentage de pauvres ne change pas beaucoup. Il y a donc forcément plus de possibilités de mobilité.

$28 T u$ as exercé, pendant plusieurs années, des responsabilités importantes au ministère de la Recherche. Tu as, dans ce cadre, largement contribué à relancer la recherche urbaine sur les pays en développement. Qu'est ce qui t'a conduit à exercer ces responsabilités? Quelles résistances as-tu rencontrées?

29 Je reviens un peu sur ma propre histoire. Comme je te l'ai dit, je suis plus un directeur de recherches ou un professeur, c'est-à-dire quelqu'un qui enseigne, que quelqu'un qui écrit. Les thèses que j'ai dirigées et mon enseignement sur les pays en développement, m'ont permis de préciser mes hypothèses de travail et d'approfondir ma propre connaissance.

Dès 1960 je pars pour une mission de coopération au Brésil pour le ministère des Affaires Étrangères français, puis dès 1965, dans le Maghreb. À partir du milieu des années 1960, je suis en position d'observateur privilégié, d'enseignant et de directeur de travaux de recherche dans plusieurs pays d'Amérique du Sud et du Maghreb. Je travaille ainsi régulièrement avec des laboratoires comme l'Institut géographique national de Rio de Janeiro ou des institutions comme l'Institut National d'Aménagement et d'Urbanisme (Inau) de Rabat. J'adapte mon enseignement à la situation et aux besoins des pays où j'interviens. Ma coopération avec l'Amérique du 
Sud et le Maghreb va se poursuivre et s'intensifier entre 1965 et 1981. Ma découverte de l'Afrique sub-saharienne commence en 1970, par le biais de l'Orstom et de ma fonction de président du comité technique d'Économie et de Démographie. J'occuperai cette fonction jusqu'en 1981.

C'est dans ce contexte que je suis nommé en 1979 à la DGRST', au comité interdisciplinaire d'études du Développement, pour m'y occuper du problème des villes et de la recherche urbaine. Et cela a été important pour moi mais pas toujours facile. J'ai présenté mes thèmes prioritaires et demandé un financement, au demeurant modeste, pour l'année 1980 : un million de francs. On ne faisait pas d'appels d'offre mais on répondait à des demandes de financement. Cette demande s'est heurtée à la très vive opposition des hauts responsables de la coopération scientifique française avec le Tiers-Monde qui considéraient ces recherches comme dangereuses dans la mesure où elles pouvaient conduire à aborder la question des luttes urbaines ce qui allait, selon eux, forcément compliquer nos relations avec les pays concernés et compromettre les relations diplomatiques entre la France et eux. De leur point de vue, il était donc hors de question que la DGRST finance des recherches qui pouvaient avoir des conséquences politiques. Il y a eu aussi des oppositions venant d'autres disciplines : la recherche agronomique sur le Tiers-Monde ne voyait pas l'intérêt que pouvaient présenter les villes qu'ils ne considéraient pas comme un élément de développement dans les pays du Tiers-Monde : laissez-les vivre et donnez-nous l'argent. « Le million que vous allez perdre pour financer des recherches urbaines, m'a alors dit un agronome de haut niveau dont j'ai heureusement oublié le nom, me permettra moi de créer un laboratoire de plus pour améliorer l'élevage bovin. »

Dans ce contexte, le changement de 1981 et l'arrivée de Jean-Pierre Chevènement au ministère de la Recherche constitue un tournant important. La décision est alors prise de créer un programme mobilisateur de recherche sur les pays du Tiers-Monde, et une commission de financement de la recherche urbaine PVD dont on me confie la présidence parce que j'étais à la DGRST depuis déjà deux ans et que j'y avais manifesté quelque humeur par rapport à la censure sur mes propositions en matière de recherches urbaines PVD. Cette commission a joué un rôle très important. J'avais les mains libres et des moyens financiers. De 1982 à 1986, entre quatre et cinq millions par an ont été alloués pour financer des travaux de recherches.

34 J'ai créé une commission où il y avait bien sûr, des représentants du ministère et de l'Orstom, dont Jean-Paul Duchemin, ainsi que des personnalités scientifiques : Philippe Hugon, Henri Coing, Xavier Godard, et d'autres. Nous avons travaillé, sans préjugés, à l'identification de thèmes de recherche sur les villes du Tiers-Monde qui nous semblaient prioritaires pour la recherche urbaine PVD. Ces thèmes étant identifiés, nous avons, dans un premier temps, attribué un premier financement, sans appel d'offre, en choisissant des laboratoires qui nous paraissaient les plus aptes à travailler sur ces thèmes. C'est seulement après un ou deux ans que nous avons lancé des appels d'offre. J'avais une grande marge de manœuvre et la confiance du ministre. Les décisions étaient prises par la commission qui n'était en réalité que consultative. En tant que président, je signais les contrats. Les deux ministres qui se sont succédés entre 1981 et 1986 nous ont toujours laissé beaucoup de liberté d'appréciation. Je n'avais pas de contraintes. On ne m'a jamais dit: "C'est une recherche dangereuse", ou " $\mathrm{Ce}$ thème ne nous semble pas important». De leur côté, les chercheurs et les équipes 
retenues avaient beaucoup de liberté pour approfondir un thème, avec les partenaires de leur choix et selon la méthode qu'ils jugeaient la mieux appropriée. Ce n'était pas toujours facile de faire accepter cette méthode de travail. Souviens-toi des difficultés que nous avons eues pour faire accepter ce mode de fonctionnement aux responsables du CEGET $^{3}$, à Bordeaux...

Quels thèmes prioritaires avez-vous identifiés?

Principalement la question du secteur informel, de l'habitat des pauvres, de la production foncière et immobilière, des transports urbains. J'ai énormément appris, d'abord par la réflexion sur l'identification de thèmes prioritaires, puis par les contacts que nous avons eus avec les équipes retenues. Cela m'a conduit a réfléchir au problèmes des villes du Tiers-Monde dans leur diversité sous un angle autre que celui de l'impérialisme et de la domination sur lequel j'avais travaillé auparavant. Je pense au travail que tu as fait sur la promotion foncière et immobilière et l'exclusion, aux recherches de Henri Coing et de son équipe sur le secteur informel, à celle de Philippe Hugon sur la petite production marchande, de Xavier Godard sur les transports urbains, pour ne prendre que ces exemples. Ces années m'ont beaucoup apporté et ont été très importantes dans mon évolution. Partant des thèmes prioritaires, je suis passé de cette expérience des différents pays du Tiers-Monde dont j'ai parlé, où je véhiculais mes propres réflexions dans mon enseignement et ma direction de travaux de recherches, à une conception beaucoup plus globale. Après 1986, mon discours qui redevient personnel est différent parce qu'il est enrichi.

Qu'est-il advenu de cette commission après 1985-1986?

Après le changement politique de mars 1986 et le retour de la droite aux affaires, cette commission a été mise en sommeil puis supprimée. La première chose que j'ai reçue, c'était une lettre me disant que, pour 1986, les financements attribués était supprimés. Heureusement c'était au mois de mars, et nous n'avions pas encore lancé d'appel d'offre. On en préparait un. Nous avons donc arrêté. Mais les programmes pluriannuels que nous nous étions engagés à financer ont été eux aussi arrêtés, faute de moyens. Tout a été bloqué. Nous nous sommes réunis encore une fois et puis au printemps 1986, la commission a été supprimée.

Quel bilan tires-tu de cette première phase 1982-1986? Combien de programmes la commission avait-elle financé? Cet appui a-t-il bénéficié à des recherches engagées dans le cadre de thèses de doctorat?

40 J'ai fait un bilan et j'ai laissé un rapport lorsque j'ai quitté mes fonctions en 1986. Au cours de ces années, nous avons, au total, financé 26 programmes et nous avons aidé à la publication de huit livres. J'ai veillé à ce que les résultats des recherches soient publiés.

41 Quant aux recherches effectuées dans le cadre de la préparation de thèses de doctorat, elles n'ont pas donné lieu en tant que telles à des financements. Cela dépendait en fait des équipes. J'ai financé des programmes de recherches collectives. Leurs responsables pouvaient très bien inclure des chercheurs engagés dans des thèses, mais cela n'apparaissait pas directement. Les crédits dont je disposais ne pouvaient être utilisés pour financer des thèses, ou d'autres travaux individuels. Certaines sans doute ont été faites grâce à ces financements.

Le travail de cette commission a-t-il eu un prolongement? 
En 1986, la rupture a été brutale. On s'est, en quelque sorte, débarrassé de moi. Une petite structure de financement de la recherche va survivre entre 1986 et 1988 pour la recherche sur les pays en développement, essentiellement sur l'agriculture, le thème urbain ayant été purement et simplement supprimé dans les programmes du ministère. Une deuxième phase va commencer en 1988, lorsque Hubert Curien devient ministre de la Recherche. Je reprends cette année-là des fonctions officielles au ministère de la Recherche pour m'occuper de la recherche sur les pays en développement. On y crée, en effet, un département de la Recherche pour le Développement, avec un comité scientifique, dont on me confie la présidence. J'ai alors surtout travaillé avec des spécialistes de l'agriculture, des agronomes et des pédologues, qui ont continué à bénéficier des financements qui leur avaient été attribués entre 1986 et 1988. J'ai beaucoup appris dans ce comité. Mon souci en tant que président a été à la fois d'assurer la continuité des programmes de recherche, principalement axés sur l'agronomie et financés entre 1986 et 1988, mais aussi de stimuler une réflexion sur d'autres thèmes prioritaires pour réintroduire la recherche sur l'urbain au sein du département. Cette initiative a reçu le soutien du ministre de la Recherche. Il réunissait régulièrement les présidents des comités scientifiques des différents départements qui l'informaient des orientations thématiques privilégiées par leurs départements respectifs et des projets de recherche en cours. Il a appuyé mon initiative de réintroduire l'urbain au sein du département de la Recherche pour le Développement. Cette initiative a également reçu un bon accueil des membres du comité scientifique pourtant peu sensibles a priori à la question urbaine.

créé un petit groupe informel pour voir quels étaient les thèmes à privilégier dans le cadre d'un possible financement en 1991-1992. S'il a fallu autant de temps pour relancer la recherche urbaine PVD, c'est essentiellement parce que la mobilisation des fonds nécessaires s'est avérée très difficile. Nous avions en effet beaucoup moins de moyens qu'entre 1982 et 1986.

46 J'ai finalement réussi, en 1992, à avoir une enveloppe pour la recherche urbaine. J'ai alors réuni une commission et j'ai demandé à un certain nombre de chercheurs, dont toi, de nous proposer des programmes à financer. Nous avons ensuite préparé et lancé les appels d'offre, au début de 1993, et examiné les réponses pour les classer et en retenir un certain nombre. Nous avons enfin informé l'ensemble des équipes retenues, en leur indiquant le montant de l'aide proposée, celle-ci devant leur être officiellement notifiée dans les semaines suivantes. Une procédure normale, en somme. Certaines de ces équipes ont même - un peu imprudemment - commencé les recherches avant de recevoir la notification officielle de la décision d'aide.

En juin 1993, avec le changement de pouvoir au sein du ministère et l'arrivée d'un nouveau responsable de la Mission Scientifique et Technique (MST) du ministère de l'Enseignement supérieur dont relevait désormais la recherche, le projet a été torpillé, ainsi d'ailleurs que tout le comité scientifique du département de la Recherche pour le développement. En quinze jours, en septembre 1993, le responsable officiel du Département, un chercheur du CIRAD ${ }^{4}$, était congédié. Pour la MST, les problèmes du développement ne semblaient pas avoir d'intérêt. Pour ma part, alors que j'avais été nommé président du comité scientifique par une lettre du ministre de la Recherche, je n'ai même pas été informé de ce démantèlement, ni reçu de lettre me disant que je n'étais plus président. Le procédé manquait pour le moins d'élégance, mais j'ai eu, surtout, le sentiment d'un énorme gâchis. 
Les équipes sélectionnées ont protesté auprès de la MST. Il ont reçu du nouveau responsable de la MST une lettre assez discourtoise qui accablait l'équipe précédente et le ministre Hubert Curien.

Le travail de la commission d'examen des réponses à l'appel d'offre dont je me suis occupé "Crise et mutation des villes dans les pays en développement " a eu un résultat paradoxal: un certain nombre d'équipes ont été retenues. D'autres, dont les projets étaient moins bons mais recevables ont été classées, avec l'espoir qu'un financement complémentaire pourrait être obtenu ultérieurement. La Caisse française de développement a alors proposé de financer ces équipes. Ce qu'elle a fait. Les premières classées ne l'ont pas été pour les raisons que tu as exposées. Ainsi, ce sont finalement les propositions qui n'étaient pas vraiment les meilleures qui s'en sont le mieux tirées... Comment vois-tu l'évolution du dispositif de recherche sur l'urbain en France? Quel est, d'autre part, ton point de vue sur la spécificité d'une recherche urbaine PVD?

Ma première observation est qu'il faut en finir avec cette idée que nous, Français, pouvons monter des programmes de recherche sur les problèmes urbains du TiersMonde en tant qu'équipe française. Je pense que maintenant, dans beaucoup de pays du Tiers-Monde, il y a des équipes et chercheurs nationaux compétents et de haut niveau, susceptibles de mener des programmes de recherche dans un cadre disciplinaire ou pluridisciplinaire. La question est donc celle du partenariat et de l'échange avec ces équipes. Une telle approche est essentielle, en particulier pour consolider nos liens de coopérations scientifiques avec les pays en développement. Il y a une volonté nouvelle de travailler dans cette direction mais il me semble que ce point de vue est moins partagé en France que dans d'autres pays européens, ou au niveau des instances communautaires. La France me parait très en retard par rapport à d'autres pays européens dans ce qui est pour moi l'avenir de la recherche urbaine. Nous manquons également de moyens pour développer des programmes de recherche conjoints avec des équipes des pays en développement, très demandeurs de cette forme de coopération à condition que ce soit dans le respect de leurs propres recherches. Les deux seuls pays où je travaille ainsi sont la Tunisie et le Brésil. Au Brésil où, après des années d'absence, j'ai fait cinq séjours au cours des 18 derniers mois, cette coopération est très appréciée. Dans ce cas particulier, c'est la partie brésilienne qui prend en charge le financement de mes missions.

51 Convient-il selon toi, comme certains le suggèrent, de mettre l'accent sur une recherche urbaine dont l'orientation serait d'abord thématique et échapperait au clivage pays développés/pays en développement?

Il nous faut plutôt partir de la demande que nous adressent les équipes et les chercheurs des pays en développement. Ce qu'ils veulent, c'est que nous leur apportions des méthodes et des outils pour mieux analyser et comprendre les problèmes urbains dans leur propre pays. Cette attente est très sensible en Tunisie ou au Brésil, pays où je travaille régulièrement. Ils ont un intérêt tout particulier pour les débats en cours, dans les pays du Nord, sur la ville et l'urbain. Quelle est l'expérience des pays développés? Quelles sont les approches innovantes, les nouveaux concepts, les nouveaux cadres théoriques? Il ont un intérêt limité pour les études de cas, les monographies, les recherches très pointues sur tel ou tel thème touchant à l'urbain. Ils peuvent les faire eux-mêmes. Ils ont, par contre, un intérêt manifeste pour les travaux de synthèse qui permettent une lecture comparée des situations prévalant, dans le Tiers-Monde, au niveau régional ou national. Dans cette perspective, il est évident que 
le clivage pays développés / pays en développement n'est pas au centre de leur préoccupation.

53 On peut s'étonner de voir des étudiants de pays lointains, venir faire, en France, une thèse sur leur pays avec les difficultés que cela comporte. Pourquoi ne pas envisager une consolidation des cycles de formation assurés dans les pays du Tiers-Monde par des universitaires français en séjour de courte durée, comme le font les Néerlandais par exemple, et non par des coopérants qui seraient là pour deux, trois ou quatre ans? La France semble réticente à mettre en place un tel système?

54 C'est exactement ma position. Je vais te donner l'exemple de ma collaboration avec le département d'Urbanisme de l'École Nationale d'Architecture et d'Urbanisme (ENAU) de Tunis au cours des six dernières années. Au départ de ce projet il y a une collaboration de la coopération française pour le programme de formation des formateurs. Des enseignants français, dont j'ai fait partie, ont été envoyés à Tunis pour des missions de courte durée destinées à aider les enseignants tunisiens pour le montage d'un DESS, puis d'un DEA. Ceux-ci sont d'abord venu soutenir leur doctorat en France. Ils peuvent maintenant le faire à Tunis. J'ai présidé en 1998 deux jurys d'habilitation à la direction de recherches pour deux maîtres-assistants tunisiens qui sont devenus professeurs. Ce programme marche bien. La France donne un appui financier significatif à ce projet. C'est exactement le cas de figure que tu évoquais.

Tu insistes sur la nécessité de développer une capacité nationale autonome d'expertise.

Exactement. Reprenons l'exemple de la Tunisie et du Brésil. Dans le premier cas, nos collègues tunisiens tiennent à développer les échanges avec nous. Il peut s'agir de chercheurs français qui viennent avec éventuellement une équipe française s'intégrant dans une équipe du pays, pour des recherches sur le pays, c'est ce qu'ils essaient de monter à Tunis. Leur centre d'intérêt reste la Tunisie.

La dynamique est un peu la même au Brésil. L'Institut Français d'Urbanisme (IFU) dont je préside le conseil d'administration est en train de monter un programme avec les Brésiliens de Fortaleza sur des problèmes urbains. Nous arrivons avec nos propres projets de recherche et notre problématique, et nous nous insérons dans ces équipes nationales tout en les aidant à se renforcer. Actuellement, ce qui les intéresse, outre leur participation aux recherches dans leur propre pays, c'est de participer au débat plus global sur l'impact de la mondialisation sur la ville dans le contexte des pays développés et des pays en développement. Progressivement, les équipes des pays en développement se structurent et s'organisent, avec nous, ou sans nous, pour comparer leurs expériences. Les échanges entre le Brésil, la Tunisie, le Maroc, la Côte d'Ivoire préparent, à terme, des réseaux sud-sud.

58 Quelles te semblent être les thématiques, les thèmes porteurs sur l'urbain dans les années qui viennent?

L'un des thèmes porteurs pour moi, c'est la gestion urbaine. Dans cette perspective, je crois qu'il faut remettre à sa juste place, le rôle de l'acteur habitant et, en particulier, ne pas surestimer l'impact de la participation des habitants sur la gestion urbaine. Je m'explique. La gestion urbaine peut, pour une large part, s'analyser en termes de jeux d'acteurs. On parle beaucoup aujourd'hui du désengagement de l'État et du rôle nouveau des pouvoirs locaux, et on ajoute que cette gestion locale repose sur la participation. On parle ainsi d'aménagement participatif, de participation des habitants à la décision, etc. Cet engouement pour tout ce qui est ou semble être "participatif » 
tend à nier - ou au moins à sous-estimer - un phénomène majeur : l'exploitation dont les individus sont l'objet. Sous couvert de participation, les pouvoirs locaux ont d'abord pour objectif de diminuer les tensions sociales, de maintenir le statu quo social tout en donnant l'illusion que les revendications de tous les citadins - y compris les groupes les plus défavorisés - sont prises en compte. La participation tend également à légitimer des pratiques qui restent fondamentalement technocratiques. Par exemple, pour quelqu'un qui travaille du lundi au vendredi, lui demander, le samedi et le dimanche, de se joindre à un groupe pour ramasser les ordures ou curer les caniveaux, n'est pas très formateur. J'ai vu ça à Madagascar. J'aurais tendance à dire que pendant ce temps, les pouvoirs locaux économisent la main d'œuvre et ne créent pas les emplois indispensables aux besoins essentiels du quartier ou de la ville.

60 Tu considères que la municipalité de Curitiba, par exemple, pratique cette forme de gestion participative?

61 Non, à Curitiba la question de la participation est abordée avec beaucoup plus de finesse. À mon avis, il existe de vraies formes de participation - et je pense que c'est largement le cas de Curitiba - qui ne repose pas sur l'exploitation de la force de travail du pauvre, mais sur une forme de participation aux décisions. Par exemple, on ne va pas aménager un quartier sans savoir ce que veulent ses habitants et comment ils le veulent. Il faut qu'ils participent à l'élaboration du programme. C'est une approche progressiste à laquelle j'adhère complètement. La participation des habitants au processus de décison peut permettre d'éviter que ne se renouvellent les erreurs du passé. Les opérations immobilières de la Banque Nationale de l'Habitat au Brésil avec leurs milliers de petites maisons toutes semblables le long des rues sont un exemple douloureux d'un processus de décision non participatif.

Mais je crois qu'il faut rester prudent sur la question de la participation pour deux raisons principales :

63 - la première, c'est la capacité réelle des populations, particulièrement des plus démunies, à concevoir leur propre espace, à expliciter ce qui serait bon pour eux, et l'extrême difficulté à le leur faire dire. Il y a ce que les sociologues appellent la stigmatisation de la pauvreté. Le pauvre a honte. Il est un humble (ce mot est horrible mais bien réel), il a une position d'humilité et il va donc être très facilement exploité, sur le plan idéologique cette fois. On peut ainsi lui faire dire ce qu'il faut qu'il dise pour justifier les pouvoirs locaux ou les intérêts des promoteurs. Il est vulnérable parce qu'il n'a pas confiance en lui. La première des choses, avant qu'il ne participe, c'est qu'il se sente citoyen ;

64 - la seconde raison (et le danger est encore plus grand) est que, sous couvert de participation, il soit manipulé par des leaders locaux plus ou moins charismatiques, des gens de son quartier qui, pour des raisons souvent inavouables vont pouvoir s'imposer. Souvent, les pauvres ne vont pas savoir se défendre, ils s'en remettent à leurs leaders qui, très souvent, dénaturent la participation des habitants et la détournent au profit de politiciens ou de partis. Il arrive souvent que les leaders trahissent ceux qu'ils sont censés représenter.

La thèse de Debora Nunes ${ }^{5}$, l'une des dernières que j'ai dirigée, porte sur un quartier pauvre de Salvador où l'université a aidé les populations démunies à monter ellesmêmes un programme d'amélioration de leur habitat avec les pouvoirs municipaux. Elle y décrit ces stigmates de la pauvreté et l'extrême difficulté qu'ont les pauvres à participer. Souvent, la participation prend la forme d'une caution donnée à des 
programmes qui ne correspondent pas à la demande et aux besoins des habitants. Ils sont trompés dans leur bonne foi.

Y a-t-il une critique à faire de ce qu'on pourrait appeler "l'idéologie participationniste" idéalisée par les organisations du système des Nations-Unies?

Je ne refuse pas la participation en tant que telle. Je pense seulement qu'elle est souvent détournée et mise au service d'objectifs qui ne sont pas conformes aux intérêts des citadins, en particulier des citadins pauvres. Peut-être que la gestion urbaine populaire ouvre des perspectives nouvelles et permettra de réels progrès. Mais pour le moment, il faut être très attentif aux dérives. On doit les analyser : quelles sont leurs formes, leurs causes, leurs conséquences et leurs acteurs? Qui en profite, les provoque et reproduit dans l'espace urbain, une fois de plus, des systèmes d'exploitation au service d'intérêts financiers ou politiques.

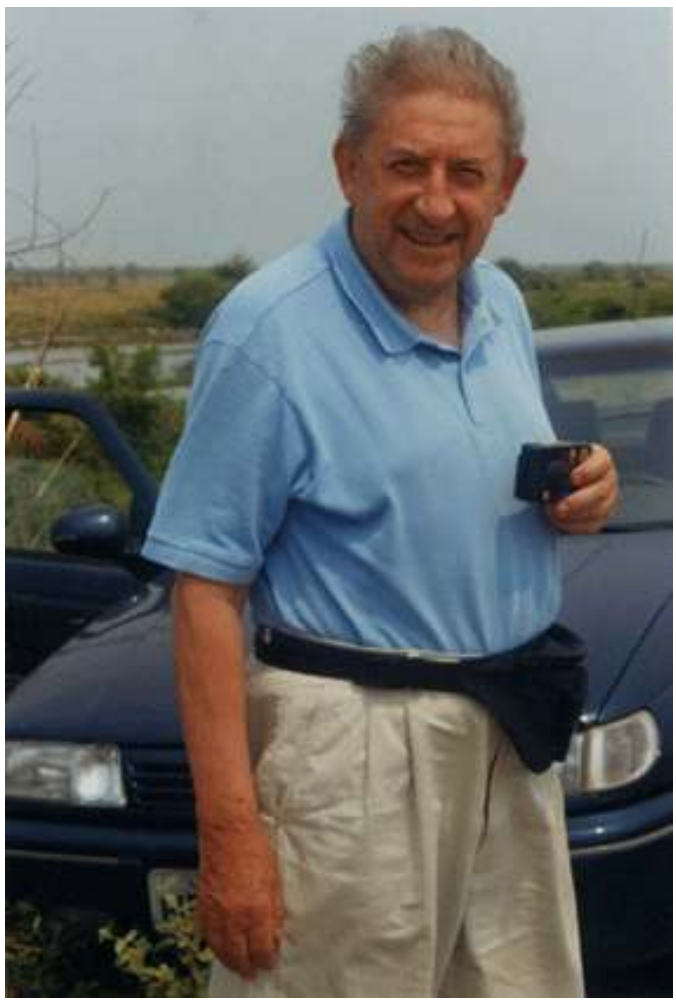

Michel Rochefort en mission dans le Pantanal (Brésil) (1999)

\section{NOTES}

1.Sabah Abouessalam, Pauvreté urbaine et comportements résidentiels à Marrakech, thèse de doctorat, université de Paris I, 1992.

2.DGRST : Délégation Générale à la Recherche Scientifique et Technique

3.CEGET : Centre de géographie tropicale, Cnrs, Bordeaux 
4.CIRAD : Centre de Coopération Internationale en Recherche Agronomique pour le Développement.

5.Debora Nunes, L'apprentissage de la citoyenneté à partir du travail communautaire. L'exemple d'un quartier populaire de Salvador, Brésil, thèse de doctorat, IUP, université de Paris 12-Val de Marne, 1998.

\section{AUTEUR}

\section{ALAIN DURAND LASSERVE}

Directeur de recherche, Cnrs 